\title{
Conformational polymorphism of a pharmaceutical cocrystal involving niflumic acid and caffeine
}

\section{Choquesillo-Lazarte ${ }^{1}$, C. Alarcón-Payer ${ }^{2}$, C. Verdugo-Escamilla ${ }^{1}$, M. Di Crisci ${ }^{1}$, J. Gómez-Morales ${ }^{1}$, A. Domínguez-Martín ${ }^{3}$}

\author{
${ }^{1}$ Laboratorio de Estudios Cristalográficos, IACT, CSIC, Granada, Spain, \\ ${ }^{2}$ Hospital Universitario Virgen de las Nieves, Granada, Spain, \\ ${ }^{3}$ Department of Inorganic Chemistry, University of Granada, Granada, Spain \\ duane.choquesillo@csic.es
}

Polymorphism in cocrystals is gaining interest because of the increasing interest in pharmaceutical cocrystals [1,2]. In this work, we report a 1:1 cocrystal of a BCS class 2 NSAID drug, niflumic acid (NIF), with caffeine (CAF) which exists in two polymorphic forms. Liquid Assisted Grinding (LAG) was used as a mechanochemical synthetic tool. Attempts to produce cocrystals by LAG led to the formation of polycrystalline material. Both the polymorphs were characterized in the solid state by diffractometric, spectroscopic and thermal methods. Recrystallization by slow solvent evaporation was carried out when the above-referred techniques strongly suggest the formation of a new solid form. In those cases where crystals were obtained, single crystal X-ray diffraction experiments were performed. Crystal structure analysis suggests that the NIF molecules in both polymorphs adopt different conformations but exhibits a common hydrogen bonding motif. Thermal analysis suggests that the polymorphs are related enantiotropically. Our work is completed with additional stability studies performed at controlled relative humidity conditions and followed by PXRD.

[1] Aitipamula, S., Chow, P. S., Tan, R. B. H. (2014) CrystEngComm, 16, 3451.

[2] Prohens, R., Barbas, R., Portell, A., Font-Bardia, M., Alcobé, X., Puigjaner, C. (2016) Cryst. Growth Des., $16,1063$.

Keywords: Cocrystal polymorphism; Niflumic acid; Mechanochemistry; Crystal structure

This research was funded by Spanish Research Agency of the Spanish Ministry of Science and Innovation, cofunded with FEDER (UE): "Bioscaffold project" grant number PGC2018-102047-B-I00 (MCIU/AEI/FEDER, UE). 